\title{
RE-LEGAZIONE E VOLONTÀ DI VERITÀ: LO SPAZIO DELLA LIBERTÀ DI FRONTE AL «POTERE DEL REALE» NEL PENSIERO DI X. ZUBIRI
}

\author{
PAOLO PONZIO \\ Università degli Studi di Bari
}

\begin{abstract}
RESUMEN: El presente artículo quiere profundizar el nexo entre voluntad de verdad y relegación en la filosofía de Xavier Zubiri, en una perspectiva diacrónica e sincrónica, en un análisis del problema del concepto de «poder de lo real» al interior del camino personal del filósofo en modo de determinar cuál es el espacio que el hombre tiene por liberarse de su fundamentalidad: agnosticismo, indiferencia y ateísmo.
\end{abstract}

PALABRAS CLAVE: Xavier Zubiri; religación; poder de lo real; agnosticismo; indiferencia; ateísmo.

\section{Re-legation and the "will of truth": the space of freedom in front of the "power of the real" in the thought of X. Zubiri}

ABSTRACT: The present article wants to deepen the relationship between the «will of truth» and the relegation in the philosophy of Xavier Zubiri, in a diachronic and synchronic perspective, in an analysis of the problem of the concept of «power of the real» in the personal way of the philosopher, in mode to determine what is the space that man has to free himself from its fundamentality: agnosticism, indifference and atheism.

KEY WORDS: Xavier Zubiri; religation; power of the real; agnosticism; indifferentism; atheism.

«Re-legazione al potere del reale, adesione intellettiva a Dio nella volontà di verità, esperienza tensiva di Dio: ecco i tre concetti che esprimono i tre momenti di un solo fenomeno, di una sola struttura, la cui unità intrinseca e formale costituisce la dimensione teologale dell'uomo, dell'uomo come costituzione dell'atto nel quale si afferma come relativamente assoluto nel seno della realtà, vale a dire, del suo Io» ${ }^{1}$.

Così si esprimeva Xavier Zubiri nel corso tenuto nell'Università Gregoriana a Roma nel 1973: tutta la questione del rapporto tra Dio e l'uomo è racchiusa nello sviluppo sistemico e integrale di questi tre concetti «filosofici». Il problema teologale dell'uomo, infatti, costituisce per il filosofo basco uno dei problemi fondamentali di tutto il pensiero filosofico occidentale, giungendo a mostrare la re-legazione come un "fatto" totale, integrale e naturale. Del resto, come più tardi scriverà in un appunto redatto a metà settembre del 1983: "siamo spinti dalle cose reali, in virtù della re-legazione, alla ricerca della realtà divina. Farsi persona è continuare a ricercare la realtà divina. La versione dell'uomo al problema di Dio è costitutiva della persona umana. Chiamerò versione teologale tale versione al problema di Dio in quanto problema» ${ }^{2}$. La re-legazione, da questo

1 Zubiri X., Hombre y Dios, (d'ora in poi HD), Alianza editorial, Madrid 2012, p. 586 (tr. it., in Zubiri X., Uomo e Dio, a cura di A. Savignano e P. Ponzio, Pagina ed., Bari 2013, p. 398.

2 HD, p. 122 (tr. it., p. 84). 
punto di vista, non è altro che il problema filosofico di Dio nell'uomo, un problema che non ha a che fare con un'ulteriorità, bensì con la formalità del reale. Di qui l'insistenza di Zubiri nel dire che la filosofia non è se non una metafisica del reale in quanto tale, intendendo la realtà nella sua formalità trascendentale 3 .

Aprirsi al problema di Dio significa, pertanto, aprirsi al tema del trascendentale senza del quale la filosofia non sarebbe che una mera costruzione di concetti e oggetti. Al contrario, proprio in funzione della sua trascendentalità originaria, il sapere filosofico può ancorarsi al reale, senza uscire da esso ${ }^{4}$ e senza costruire il suo oggetto: è la stessa realtà che si offre alla conoscenza filosofica attraverso quello che Zubiri chiama il «potere del reale». Ecco dunque profilarsi quali siano i passaggi fondamentali per poter cogliere, da una parte, l'evoluzione del termine re-legazione all'interno della vicenda intellettuale del filosofo e, dall'altra, determinare tutti quei passi decisivi che portano l'uomo a tentare di «liberarsi» dal proprio fondamento, esprimendo nell'ateismo la propria «volontà di fondamentalità».

\section{RE-LEgAZIONE: STORIA DI UN CONCETTO, STORIA DI UNA VICENDA PERSONALE}

Quantunque sia nell'articolo En torno al problema de Dios ${ }^{5}$, che Zubiri presenta per la prima volta il termine "religación» (re-legazione), la sua idea era già stata introdotta in nuce nel saggio Filosofía y Metafisica, pubblicato nella Revista de Occidente nel 1935, laddove si parla della forza delle cose che si impongono all'uomo dettandoci il loro essere ${ }^{6}$. L'importanza di questo primo contributo è data dal fatto di situare il problema di Dio in quello che era allora il nucleo centrale del pensiero contemporaneo, vale a dire, la filosofia dell'esistenza, così da indirizzare la questione teologica all'interno di una prospettiva meramente antropologica. Il problema concernente Dio deve essere così ricondotto alla questione concernente l'uomo. Ed è a partire da questo contesto esistenziale che Zubiri si pone una serie di domande che presuppongono una sua apertura al pensiero metafisico, un suo passaggio dall'ontologia ermeneutica di matrice heideggeriana alla metafisica. Pertanto, la domanda su Dio si tradurrà

3 «La filosofía no es filosofía ni de la objetividad ni del ente, no es fenomenología ni ontología, sino que es filosofía de lo real en cuanto real, es metafísica»: Zubiri X., Naturaleza, Historia, Dios, Alianza Editorial, Madrid $1994^{10}$, p. 16 (tr. it., Edizioni Augustinus, Palermo 1985, p. 21).

4 «Il problema della ragione non consiste nel verificare se è possibile che la ragione giunga alla realtà, ma proprio il contrario; in che modo occorra mantenerci nella realtà nella quale già stiamo»: ZuBIRI X., Inteligencia y razón, Alianza editorial, Madrid 1982, p. 96.

Larticolo si trova ora in ZuBIRI X., Sobre el problema de la filosofía y otros escritos (1932-1944), Alianza Editorial - Fundación Xavier Zubiri, Madrid 2002, pp. 215-241. Lo stesso filosofo spagnolo ha rifuso il suo articolo in Naturaleza, historia, Dios, o.c., pp. 417-454 (tr. it., pp. 249-271).

6 «las cosas arrastran al hombre por su ser. El hombre dice lo que dice por la fuerza de las cosas»: Zubiri X., «Filosofía y metafísica», in Revista de Occidente, 1930, p. 46. 
nelle seguenti questioni: «Qual è la relazione dell'uomo con la totalità della sua esistenza? Qual è il carattere di questo esser gettato tra le cose? È soltanto un "semplice" trovarsi o qualcosa di più?» ${ }^{7}$.

Zubiri sa bene che si potrà "superare» la filosofia heideggeriana solo a partire da essa. Qualsiasi tentativo che non si muovesse dalla stessa prospettiva esistenzialista, sarebbe destinato a naufragare nei gorghi della fatticità. Come giustamente rileva Oscar Barroso, «la strategia è incontrare nella fatticità qualcosa che vada più in là della stessa fatticità ${ }^{8}$. E a ragione Diego Gracia rileva che è qui dove Zubiri dialoga maggiormente con Heidegger, riscrivendo l'heideggeriano Geworfenheit in una prospettiva di approfondimento dell'esistenza umana fuori da se stessa:

«La religación es otra cosa, el análisis y descripción de una experiencia fundamental, de un dato primario. Esa descripción de una experiencia fundamental, de un dato primario. Esa descripción de una experiencia la hace Zubiri, sin duda, tomando como base los análisis heideggerianos de la Geworfenheit. Pero Zubiri pretende ir más allá, profundizar en el carácter yectivo de la existencia humana (...) El ser humano está yecto desde, y por tanto se halla "puesto por atrás o desde atrás" en la existencia. En la religación el hombre no tiene la iniciativa»?.

Il problema si situa, pertanto nel chiedersi se esista un dato più originario dello stesso factum dell'esistenza umana, se cioè la fatticità dell'esistenza sia solo una denominazione provvisoria o vi sia un qualche apoyo che sostenga l'uomo nell'esistenza, un qualche dato esterno all'uomo che sa essere:

"Vale a dire che l'uomo, nell'esistere, non solo si trova con le cose che "ci sono" e con cui deve farsi, ma si trova anche con il fatto che "bisogna" farsi e "deve" starsi facendo. Oltre alle cose, "c'è" anche ciò che fa sì che esse ci siano» ${ }^{10}$.

E questo far sì che le cose e l'esistenza stessa ci siano, altro non è se non qualcosa di più radicale della stessa fatticità che non può spiegarsi in una semplice manifestazione di obbligazione a esistere. Anzi, l'obbligo stesso non è che la conseguenza di qualcosa di più originario: «siamo obbligati a esistere, perché siamo previamente re-legati a ciò che ci fa esistere» ${ }^{11}$. La re-legazione è dunque un "vincolo ontologico» primordiale rispetto a ogni altro carattere esistenziale. Tale vincolo assume, poi, alcuni caratteri specifici che Zubiri opportunamente rileva.

7 Zubiri X., Naturaleza, Historia, Dios, o. c., p. 424 (trad. it., p. 254).

8 Barroso Fernández O., «La existencia en la filosofía española del siglo XX: Unamuno y Zubiri», in "L'esistenza», a cura di Esposito C. e Carraud V., in Quaestio. Annuario di storia della metafisica, 3, 2003, pp. 371-390 (in part. p. 382).

9 Gracia D., "Zubiri y la experiencia teologal. La difícil tarea de pensar a Dios y la religión a la altura del siglo XX", in Filosofía como pasión. Homenaje a Jorge Eduardo Rivera Cruchaga en su 75 cumpleaños, a cargo de P. Brickle, Trotta, Madrid 2003, pp. 249-263, (in part. pp. 252-253).

10 Zubiri X., Naturaleza, Historia, Dios, o.c., p. 428 (trad. it., p. 256).

11 Ibid. 
Innanzitutto il vincolo della re-legazione non è qualcosa di esterno all'essere stesso dell'uomo. Nella re-legazione, infatti, l'uomo si trova vincolato a qualcosa che previamente ci fa essere, da cui veniamo all'essere. In questo modo il relegare non è mai un andare verso qualcosa ma, al contrario, un venire $\mathrm{da}^{12}$. Questo vincolo appartiene così non tanto alla sfera dell'avere ma a quella dell'essere: «L'uomo non ha una religione, ma piuttosto velis nolis, consiste in re-legazione o religione» ${ }^{13}$. Da questa angolazione non vi è differenza tra un teista e un ateo, poiché, quantunque negata —o nascosta, come suggerisce Zubiri- l'uomo non può perdere se non apparentemente questa fondamentalità dell'esistenza scoperta.

La condizione della re-legazione, infine, vincola il problema ontologico verso una nuova strada che sarà percorsa dal filosofo negli anni successivi la pubblicazione di Naturaleza, historia, Dios, e che viene qui introdotta nelle sue indicazioni essenziali: la dialettica ontologica non pone soltanto il problema di ciò che c'è, intendendolo come essente, bensì pone il problema di ciò che «fa che ciò ci sia». L'intelletto non trova soltanto che le cose ci sono, ma che c'è pure ciò che re-lega l'esistenza. Il problema filosofico di Dio non è, allora, qualcosa di differente dalla domanda sull'essente, anzi dipenderà soltanto «dalla possibilità di includerlo nell'è» ${ }^{14}$. Non si tratta di un ampliamento, ma di un'inclusione possibile soltanto perché Dio ha già qualcosa a che vedere con l'essere, per il fatto che sono le cose che ci sono ${ }^{15}$. Ed è proprio nella comprensione che si compie lo smarcamento dalla fenomenologia classica di marca husserliana e heideggeriana, giungendo a quella prima grande intuizione zubiriana che consisterà nel dare centralità al «sentire» in quanto esperienza originaria delle cose. Secondo il filosofo spagnolo, Heidegger è stato incapace nell'andare più in là del «senso dell'essere»: il suo concetto di esistenza, infatti, è puramente modale, a differenza di quella che Zubiri intravvede essere il significato originario del termine exsistentia, che non designa una «modalità» quanto una «realtà», quella di sussistere al di fuori delle cause, vale a dire, quella di essere «realmente» persona ${ }^{16}$. E sarà grazie a questa intuizione che Zubiri inizierà a

12 "Se si vuole, è un "andare", ma un andare che consiste non in un "compiere" ma in un mostrare rispetto a ciò da cui veniamo, "essere chi già sei" ». Zubiri X., Naturaleza, historia y Dios, o.c., pp. 428-429 (trad. it., p. 256).

13 Ibid., p. 430 (trad. it., p. 257).

14 Ibid., p. 440 (trad. it., p. 263).

15 "Cioè, non è che da un lato stia l'esistenza umana e dall'altra Dio, e che "dopo" si tenda il ponte dal quale "risulti" che Dio fa sì che ci sia l'esistenza. No: il modo primario in cui (se si vuole usare l'espressione) per l'uomo "c'è" Dio, è il fondamentare stesso; meglio ancora: dal punto di vista umano, la deità è lo stare fondamentando». Ibid., p. 441 (trad. it., p. 263).

16 Cfr. Conill J., «Heidegger y Zubiri», in Revista Portuguesa de Filosofia, 59, fasc. 4, 2003, pp. 1181-1202 (in part. 1191): "La estructura heideggeriana es un concepto modal, hace referencia a lo que el hombre hace de si. Sin embargo, Zubiri no puede olvidar que en su acepción primitiva, la que le dio Ricardo de San Victor, exsistentia no designaba una "modalidad" sino una "realidad", la de subsistir fuera de las causas (sistere extra causas), es decir, la de ser "realmente" persona». Cfr. anche Gracia D., "La historia como problema metafísico», in Realitas, III-IV, pp.79-149. 
separarsi dalla fenomenologia, attuando quel percorso differente che lo condurrà a porre le basi della sua filosofia dell'intelligenza.

Nel 1963 Zubiri torna a occuparsi del concetto di re-legazione nel saggio Introduccion al problema de Dios, pubblicato nella quinta edizione di Naturaleza, Historia, Dios. L'intento del filosofo è quello di cercare una nuova sistematizzazione del problema, accentuando ancor di più la valenza della persona. L'uomo non è una cosa tra altre, ma una realtà strettamente personale: non si tratta di cosa o come l'uomo agisca, di quali siano i suoi atti, ma del suo posto nella realtà.

«Este carácter fundante hace que el hombre en sus actos no sea solo una realidad actuante en una $\mathrm{u}$ otra forma, sino una realidad religada a la ultimidad. Es el fenómeno de la religación. La religación no es sino el carácter personal absoluto de la realidad humana actualizado en los actos que ejecuta. El hombre está religado a la ultimidad porque en su propia índole es realidad absoluta en el sentido de ser algo "suyo" »"

Il costituirsi della re-legazione passa, così, attraverso il carattere personale assoluto della realtà umana: l'uomo è re-legato all'ultimità poiché nella sua natura è realtà assoluta, realtà «da sé», ma il suo assoluto è relativo perché l'essere dell'uomo deve irrimediabilmente compiere la sua personalità fondato nella realtà stessa. Cos'è questa ultimità? Nella re-legazione, spiega Zubiri, «veniamo re-legatamente da una ultimità, da una deità». È evidente che qui Zubiri sceglie deliberatamente di non utilizzare il termine Dio per sottolineare una anteriorità dell'idea di deità rispetto a quella di Dio, sicuramente ispirandosi, come Gracia attesta, all'idea heideggeriana della Göttliche ${ }^{18}$. E tuttavia, mentre nel filosofo di Friburgo l'anteriorità della Deità serve per «neutralizzare fin da principio ogni possibile riferimento alla Trinità ${ }^{19}$, vale a dire, per neutralizzare qualsiasi riferimento al Dio cristiano - sempre accuratamente evitato da Heidegger- in Zubiri il ricorso alla deità rappresenta la condizione per non dare per pre-supposta la realtà di Dio.

Anzi la deità è un'esperienza propria dell'atto personale della re-legazione che rende attuale il potere del reale. Pertanto, la deità non è se non la stessa realtà in quanto potente e re-legante, per cui ciò che caratterizza la deità è proprio il suo potere. Un potere che ha diversi aspetti, come spiegherà nel corso su Il problema filosofico della storia delle religioni nel 1965: è un potere trascendente, vivo e vivificante, che conforma ogni singola cosa separandole, un potere dell'intero ciclo germinale, un principio di organizzazione degli esseri viventi, un potere della promozione dei risultati, un potere che vincola gli uomini tra loro, un potere che domina i due fatti polari dell'esistenza degli esseri viventi,

17 Zubiri X., Naturaleza, Historia, Dios, o. c., p. 411.

18 Gracia D., Religación y religión en Zubiri, in Filosofía de la religión, a cargo de M. Fraijó, Tecnos, Madrid, 1994, p. 499.

19 Strummiello G., "Got(t)heit: la Deità in Eckhart e Heidegger», in C. Esposito e P. Porro (a cura di), Heidegger e $i$ medievali, a cura di Esposito C. e Porro P., in Quaestio, 1 (2001), pp. 339-359, in part. p. 355. 
la vita e la morte, un potere che si manifesta nelle diverse vicende umane, un potere del destino degli uomini, un potere dell'unità del cosmo, un potere del sacro, un potere delle virtù personali, un potere che riempie il tutto e un potere che perdura in eterno ${ }^{20}$.

Tutti questi aspetti sono segno del fatto che l'uomo nella re-legazione non perviene alla deità, ma al contrario è qualcosa da cui si viene. Ed è un'apertura che non è il risultato di un carattere morale, sentimentale o psicologico né, tantomeno, dipende da una struttura sociale, bensì tali aspetti sono tutti presenti nella deità in quanto re-legazione. Così come l'atto re-legante non è un atto particolare, o addirittura, come potrebbe dirsi, l'atto per eccellenza dell'essere umano: bensì «il carattere che ogni atto ha per poter essere atto di una realtà personale ${ }^{21}$. Questo è il motivo per cui la re-legazione non ha una «origine» ma un "fondamento», ed è compito dell'intelligenza poterlo mostrare. Un'intelligenza che è obbligata dalla stessa realtà a porsi il problema della deità, del fondamento ultimo del reale, giungendo a esprimere la deità in quanto realtàdeità ${ }^{22}$. Ma in che modo l'intelligenza può conoscere la deità? Per il carattere remittente della stessa realtà-deità che è «causa prima» del carattere della deità e di tutta la realtà, una realtà-deità che, se si vuole, può anche essere denominata realtà divina, una realtà che sta fondando il mondo come realtà al di fuori del mondo. È la «realtà trascendente assoluta»»33 la quale per essere causa delle realtà umane deve possedere in modo eminente i caratteri dell'intelligenza $\mathrm{e}$ della volontà: "questa realtà trascendente della causa prima è una realtà intelligente e volente» ${ }^{24}$.

«Deidad, realidad primera, realidad personal y libre, esto es, deidad, realidad divina, Dios: he aquí los tres estadios del descubrimiento intelectivo de Dios. Cada uno de ellos se apoya en el anterior y conduce por interna necesidad al siguiente $»^{25}$.

Deità, realtà-deità sono espressioni che Zubiri cercherà di precisare meglio durante i corsi degli anni '70 e soprattutto nella redazione finale de L'uomo e Dio nel 1983, ridefinendone i contorni gnoseologici e metafisici, portando il filosofo ad assumere come più indicativi termini quali realtà-fondamento e potere del reale e lasciando al lemma «deità» una connotazione di manifestazione formale di Dio nelle cose, a parte dei.

20 Cfr. la nuova edizione de El problema filosófico de la historia de las religiones (1965), in ZuBIRI X., Sobre la religión, a cargo de Gonzalez A. e Vargas E., Alianza Editorial, Madrid 2017, pp. 79-86. Si veda anche ZuBIRI X., El problema filosófico de la historia de las religiones, Alianza editorial, Madrid 1993, p 51.

21 Zubiri X., Naturaleza, Historia, Dios, o.c., p. 411.

22 Cfr. Ibid., pp. 411-412.

23 Ibid., p. 413.

24 Ibid., p. 413.

25 Ibidem, p. 414. 


\section{IL POTERE DEL REALE E LA SUA CONOSCENZA}

Le cose veicolano la realtà che, assumendo il carattere di fondamento-attraverso i tre aspetti di ultimità, possibilità e impellenza-, mostra il suo duplice significato: da un lato si configura come radicale alterità rispetto all'uomo, dall'altro è ciò che permette la realizzazione personale. Di qui l'emergere di domande sul fondamento che non è «causa» bensì «dominio» del reale, un potere della rispettività del reale. Il "potere del reale» si impossessa così dell'uomo, dominandolo ${ }^{26}$. È il ribaltamento della prospettiva soggettivistica della modernità: non è più l'uomo a imporsi sul reale, ma ciò che domina è la realtà: una posizione questa che assume un profilo non è meramente gnoseologico visto che il legame al «potere del reale» costituisce, dal punto di vista metafisico, ciò che Zubiri chiama re-legazione, concepita come un fatto totale e radicale il cui oggetto formale è la natura personale, l'unica in grado di attualizzarsi. Totale perché concerne tutta la realtà personale, radicale poiché è la re-legazione alla realtà che fonda la persona.

La re-legazione si configura, pertanto, come manifestazione del potere del reale, un'esperienza che comprova l'effettiva realtà di qualcosa, ed è una dimensione formale della persona umana o, se si vuole, un fondamento per e dell'essere. Non si tratta, dunque, di un influsso sull'uomo da parte del potere del reale, che si attua in un determinato momento della vita, quanto di un «dominio" costitutivo e intrinseco: l'uomo per essere se stesso, per essere reale, viene da esso. Il potere del reale ci colloca nella realtà e ci lega a essa. Ed è un collocamento che si attua non andando verso la realtà ma procedendo da essa, così come il legame al reale ci costituisce come ab-soluti, cioè «liberi» dinanzi al reale: paradossalmente è proprio legandosi al potere del reale che l'uomo vive pienamente il suo essere relativamente assoluto.

E tuttavia il potere del reale è anche una forma di concettualizzazione ${ }^{27}$ per il fatto che la realtà è presente in ogni apprensione umana, come in un vincolo indissolubile tra intelligenza e realtà: l'impressione di realtà è dotata «de un carácter insondable y poderoso», come si legge in Intelligenza senziente ${ }^{28}$.

Il vincolo con la realtà non è un semplice riferimento alle cose nell'intelligenza: Zubiri per spiegare tale nesso dovrà formulare un nuovo concetto con il quale far cogliere al lettore tutta la portata di tale implicazione. L'attualizzazione della realtà nell'intelligenza senziente svolgerà proprio tale compito, rendendo presente la realtà nell'intelligenza, una presenza ineludibile e inalterabile: una presenza che resta nell'atto che apprende e che rappresenta l'origine di ogni possibile atto conoscitivo. Per spiegare questo

26 «El poder de lo real se apodera de mí. Y gracias a este apoderamiento es como me hago persona» HD, p. 95 (tr. it., p. 64). 169-172.

27 Cfr. Díaz C., «El hombre y Dios en Xavier Zubiri», in Revista Agustiniana, 34 (1993)

28 Cfr. ZuBIRI X., Inteligencia sentiente. Inteligencia y realidad, Alianza editorial, Madrid 1991, p. 64. 
aspetto, il filosofo ricorrerà al termine «noergia»: «lo radical es un devenir de actualidad, un devenir que no es noético ni noemático sino noérgico ${ }^{29}$. È uno «stare» della realtà in quanto tale nell'apprensione che fonda qualunque momento ulteriore e qualunque altro modo di apprensione: «la noergia, en cambio, no es mera ratio cognoscendi sino la realidad misma en su imponente actualidad: es la ratio essendi de lo real $»^{30}$. Quando apprendiamo il reale, la realtà supera sempre trascendentalmente il suo contenuto concreto, precede il suo contenuto specifico, e la noergia altro non è se non questa precedenza della realtà nell'ordine della realtà stessa. Questo carattere noergico dell'intellezione rende possibile il potere del reale: «las cosas reales no consisten tan sólo en la intrínseca necesidad de la estructura de su contenido y de la fuerza con que este contenido se nos impone según su formalidad; consiste también en vehicular trascendentalmente el poder mismo de lo real, la dominancia de la formalidad sobre el contenido ${ }^{31}$. Il momento trascendentale è la forza della realtà, ma tale momento è sempre "più» del momento talitativo della realtà stessa, sempre "più» del suo contenuto: una precedenza rispetto alle singole cose reali e, insieme, una domanda di fondamento. Il potere del reale, al pari di ogni fatto attualizzato nell'apprensione, richiede che si vada al fondamento dell'apprensione stessa, al fondamento dell'atto appreso.

All'interno di questa formalità di apertura trascendentale quale realtà viene veicolata? Nel mio approccio conoscitivo della realtà nel suo aspetto enigmatico, qual è il carattere proprio della realtà che mi si presenta? Non è certamente la realtà in quanto cosa, la "realtà-oggetto», quella che mi si presenta nella sua enigmaticità (un tavolo sarà sempre una realtà-oggetto), bensì quella che Zubiri denomina la «realtà-fondamento». È questa la realtà che si presenta a me e verso cui ogni uomo è inesorabilmente lanciato; e questo per due motivi: innanzitutto per il fatto che ogni uomo è orientato non verso le cose o di fronte a esse, ma "verso» la realtà-fondamento; in secondo luogo, per il fatto che ogni uomo è sempre di fronte a un'opzione: lanciato «verso» la realtà-fondamento deve optare per una determinazione della realtà, per una forma di realtà.

Lanciato inesorabilmente verso la fondamentalità della realtà, costretto -in qualche modo- dal potere del reale, l'uomo non perde la sua libertà: la volontà di aderire o meno, di re-legarsi a tale fondamentalità, a tale originalità del reale, a tale trascendenza del reale.

Ma come è possibile per l'uomo accedere a tale fondamentalità?

Ogni conoscenza è sempre, secondo Zubiri, un'attualizzazione di ciò che è intelligito nell'intelligenza e pertanto la funzione di quest'ultima dipende dalla natura di ciò che si sta attualizzando. Non è la stessa cosa conoscere la realtà nella sua oggettualità e nella sua fondamentalità. Mentre, infatti, nel primo caso la funzione intellettiva giunge alla scoperta dell'oggetto nella sua inseità, nel

29 Ibid..

30 SOlari E., La raíz delo sagrado: Contribuciones de Zubiriala filosofía dela religión, Ril editores, Chile, 2010, p. 139.

31 ZubiRI X., Inteligencia sentiente. Inteligencia y realidad, cit., p. 198. 
secondo caso la stessa intelligenza è chiamata a un compito più complesso: alla funzione di scoperta della realtà si aggiunge quello della fondamentalità che è l'ambito attraverso cui si rende possibile che la mia realtà giunga al suo fondamento. Conoscere la realtà-fondamento — cioè, conoscere Dio, realitas fundamentalis - significa attualizzare una realtà non come oggetto presente in sé, ma come realtà fondante, una presenza che apre l'ambito della fondamentalità del mio io e rende possibile tale conoscenza, rende possibile, cioè, l'adesione al fondamento del mio io. La conoscenza della realtà-fondamento, dunque, costituisce già la possibilità della fede. In questo modo Zubiri cerca di superare le concezioni classiche del rapporto fede e ragione concepite sempre all'interno di una dicotomia originaria tra il credere e il conoscere, indicato attraverso il primato ora dell'uno, ora dell'altro dei termini ${ }^{32}$. Ciò che, invece, preme al filosofo spagnolo è indicare un'unità più originaria e radicale: l'unità tra conoscenza e ambito di adesione nell'intellezione di Dio come realtà-fondamento. E tale unità radicale si esprime nei termini di una possibilità.

Di che si tratta? La possibilità riguarda non solo la realtà in quanto tale, ma anche il modo attraverso cui la realtà possibile viene attualizzata dalla mia intelligenza: le cose reali possibili mostrano, così, anche l'ambito delle mie possibilità reali. Il reale possibile chiede di prendere posizione, sollecita un'adesione della realtà personale a una determinata possibilità. È ciò che Zubiri chiama atto di appropriazione, un atto attraverso cui l'uomo decide di una possibilità come modo del proprio essere. L'ambito della possibilità, allora, è tout court l'ambito della volontà: è volontà di essere, o meglio, volontà di verità.

Tale volontà è in piena coerenza con l'essenza dell'intelletto che cerca la verità nella piena manifestazione, fedeltà ed effettività. Zubiri ci avverte del pericolo di considerare la verità reale come un semplice innesco del processo intellettivo. «(...) la verità reale non è solo l'inizio di un processo intellettuale ma il principio di ogni atto di intelligenza in questo processo» ${ }^{33}$. E proprio perché principio, è presente nell'intero processo in modo attuale, «(...) ogni atto di un processo intellettuale è fondato sulla presenza stessa della verità reale» ${ }^{34}$, anche, naturalmente, nell'appropriazione di possibilità.

È importante sottolineare qui l'unità e l'attualità nella persona di tutto il processo che si sviluppa: la persona non è una realtà data una volta per tutte ma è in un continuo processo di realizzazione del suo essere. L'intelletto e la volontà sono correlati nella realtà personale come auto-possesso di sé. Ciò significa che non possono essere intesi separatamente al di fuori di questa realtà e dal dinamismo del «da sé». E proprio perché l'intelligenza è mia, allora tutti i suoi atti sono miei come persona. Allo stesso modo, per il fatto che la volontà è mia anche tutti i suoi atti sono miei e della mia intelligenza. La realtà personale

32 Il credo ut intelligem o l'intelligo ut credam, infatti, secondo Zubiri, sottolineano un'unione tra fede e conoscenza in termini di passaggio: «la fede come cammino per la conoscenza, o la conoscenza come cammino per la fede». HD, p. 253 (tr. it., p. 173).

33 HD, p. 262 (tr. it., p. 178).

34 Ibid. 
è l'attualità di tutta la sua realtà in quanto tale. Intelligenza e volontà, verità e ambito di possibilità, acquisiscono realtà nella realtà personale. Da questo punto di vista, dobbiamo comprendere la riflessione sull'unità dell'intelligenza e della fede, dell'intelligenza e della volontà. È tutta la realtà personale nell'attualità radicale che si consegna, che crede e che attua. È tutta la realtà personale attuale che accede a Dio negli atti che esegue.

La verità reale, vale a dire l'attualizzazione della realtà nell'intelligenza, ci lancia a «ideare», ci apre a due nuovi ambiti di possibilità reale: da un lato vi sono le idee delle cose in e per se stesse, dall'altro la possibilità di dirigersi verso la realtà stessa, considerando le idee come organi che rendono di volta in volta più presenti le cose. L'uomo tra queste due possibilità deve optare, deve compiere un atto di volontà, una volontà di verità: volontà di verità delle idee, se si opta per la prima possibilità, volontà di verità reale, se si sceglie la seconda via. Inutile dire che in Zubiri appare sempre molto forte la critica a ogni possibile concettualismo che dimentica la realtà o la riduce a un mero e inutile incidente gnoseologico. Invece la "volontà di verità reale comporta più presenza di realtà nell'uomo. Perciò la volontà di verità reale è la condizione precisa per il conseguimento di verità reali » ${ }^{35}$. Nella misura in cui opto per la volontà di verità, mi approprio e realizzo le mie possibilità. È un'opzione resa possibile dalla verità reale in quanto attualizzazione della realtà nell'intelletto. La verità reale precede la volontà di verità reale e rende l'opzione possibile all'uomo, implicando il mio stesso essere. Ogni appropriazione è incorporazione reale delle possibilità date alla mia personalità: «La volontà di verità reale implica il mio essere non come un'espressione autentica di questi, ma configurandolo per l'appropriazione delle possibilità che la verità reale offre ${ }^{36}$. Non è un'espressione del mio essere altrimenti io potrei ergermi a origine della verità: è, invece, una configurazione di me stesso per le possibilità della verità che mi precede.

"Nell'adesione alla verità reale si realizza "unitariamente" la presenza di realtà e la realizzazione del mio essere nell'adesione a quella verità ${ }^{37}$. Adesione, presenza della realtà e realizzazione incontrano la loro unità nella persona. La realtà personale non è solo il luogo attraverso cui la verità si manifesta o meno, ma anche dove le possibilità offerte da questa verità diventano efficaci e si stabiliscono configurando la persona. Questo continuo riferirsi alla realtà personale è indispensabile poiché è solo in essa che l'adesione non induce a un annichilimento bensì ad uno sviluppo. L'adesione radicale nella fede alla realtàfondamento non suppone alcuna diminutio nella persona ma, al contrario, la configurazione nella pienezza del suo proprio essere sostantivo. Tuttavia, non possiamo dimenticare che ciò che media tra la verità e la volontà di verità reale è la volontà dell'uomo che opta per le possibilità che gli vengono date. Possibi-

\footnotetext{
35 HD, p. 264 (tr. it., p. 179).

36 Ibid.

37 Ibid.
} 
lità che dipendono dai modi secondo cui la realtà si fa presente: se, infatti, la realtà si fa presente come realtà-oggetto, la verità reale è l'attualizzazione della realtà in sé e per sés ${ }^{38}$. Ma la realtà può essere presente anche come realtà-fondamento, nella quale io stesso sono momento di attualità: la realtà-fondamento è realtà in sé e per sé, ma lo è attraverso di me: «il modo stesso di essere presenza di questa realtà è "presenza-per" me» ${ }^{39}$. Per questo motivo, l'attualizzazione secondo la realtà-fondamento costituisce una possibilità «mia», del mio essere sostantivo, del mio Io. E dato che si tratta di possibilità, si aprono — secondo Zubiri-due possibili strade: essere termine di un lasciare che la propria realtà sia ciò che è in sé e per sé, o essere termine di un lasciare che fa sì che accada in me tale realtà fondandomi in quanto realtà ${ }^{40}$. Quest'ultima è possibilità di fondamentalità, mentre la prima è possibilità di lasciare in sospeso tale fondazione, vale a dire, riduzione della realtà a mera realtà-oggetto. L'uomo opta per la fondamentalità, sceglie la possibilità che la fondamentalità della realtàfondamento accada nel mio essere, vale a dire costituisca l'attualità della fondamentalità in me. E, così, spiega Zubiri:

«La verità reale della mia persona, nelle sue tre dimensioni di manifestazione, fedeltà e irresistibile effettività, si trova configurata nella verità reale della realtà-fondamento: la mia manifestazione come essere relativamente assoluto è la manifestazione dell'assoluto del fondamento; la mia fedeltà è fedeltà nell'assoluto; la mia effettività è l'irresistibile effettività nell'assoluto. Allora la volontà di verità reale è volontà di fondamentalità ${ }^{41}$.

L'adesione alla realtà-fondamento implica il fatto di dover scegliere per la fondamentalità stessa, significa fare mia la fondamentalità. La volontà di fondamentalità ci spinge a conoscere la verità nella sua realtà-fondamento: essa non è un atto consecutivo al processo di intellezione, bensì un'attitudine che da il primo impulso al processo intellettuale: «Tale volontà è, pertanto, come ogni volontà di verità, una volontà antecedente in qualche modo alla verità intellettiva. Tale «antecedenza» non è un atto. Se così fosse, si potrebbe pensare che è una volontà che preme perché il conosciuto mi appaia previamente ciò che vorrei che fosse ${ }^{42}$. La volontà di fondamentalità, come ogni volontà di verità non è atto ma attitudine, perché indica l'attitudine di adesione del mio essere a ciò che si mostra presente come fondamento.

38 Tutto il sapere scientifico, secondo Zubiri, si poggia sull'appropriazione della possibilità di un'adesione alla realtà tramite la realtà stessa: «Si tratta, tra l'altro, dell'origine della scienza (nel senso più ampio del termine). Il sapere scientifico, qualunque esso sia, deve la sua esistenza alla volontà di verità reale. È un'opzione libera: potrei aver scelto per aderire non alla realtà tramite la realtà ma in vista dell'utilizzazione, applicazione, ecc. (poco importa ora il termine) delle realtà». HD, p. 265 (tr. it., p. 180).
39 HD, p. 266 (tr. it., p. 181).
40 Cfr. HD, p. 267 (tr. it., p. 182).
41 HD, p. 269 (tr. it., p. 183).
42 HD, p. 271 (tr. it., p. 184). 
Tuttavia, come spiega in seguito Zubiri, la volontà di fondamentalità in quanto attitudine non è soltanto impulso del processo intellettivo, bensì si esprime anche nella volontà di accettazione della realtà-fondamento: è «l'attitudine di adesione al fondamento scoperta dalla ragione ${ }^{43}$. Una ragione che non indica necessariamente una verità sufficientemente provata, ma che è conforme alla ragione: una congruenza tra razionale e ciò che è voluto. È la ragione che mostra ciò che è ragionevole indipendentemente dalla sufficienza di tale conoscenza: da questo punto di vista il ragionevole è più che razionale, «è il razionale trasfuso in tutto l'essere dell'uomo» ${ }^{44}$.

Come attitudine, poi, la volontà di fondamentalità, in quanto libera e ragionevole, è principio di adesione reale a ciò che l'intelligenza conosce. Grazie a questo l'adesione da essere una semplice possibilità costitutiva della conoscenza della realtà fondante diventa realizzazione libera di quella possibilità ${ }^{45}$. Una possibilità che si gioca nello spazio della ragione che è sempre un luogo di ricerca, un luogo che cerca il fondamento, all'interno delle "possibilità» costitutive della stessa realtà. Naturalmente la risposta può ricorrere a vie molto differenti, come ci mostra la storia delle religioni, una storia intesa come l'esperienza storica, individuale e sociale dell'umanità in cerca del fondamento ultimo del potere del reale, che le religioni denominano con il termine di Dio. All'interno di tale problema ricadono anche le risposte che riguardano una certa «indifferenza intellettiva» per le quali si sospende ogni processo dell'intelligenza che guardi al fondamento del reale ${ }^{46}$, o quelle negative per le quali non vi è altro fondamento del potere del reale se non la fatticità stessa della vita, una vita a-tea ${ }^{47}$.

\section{UNA VOLONTÀ SOSPESA: IL CASO DELL'AGNOSTICIMO E DELL'INDIFFERENTISMO}

«Che la volontà di verità, come volontà di fondamentalità, ponga in cammino il processo intellettivo è qualcosa che appartiene intrinsecamente a tale volontà. Ma non è detto che il processo intellettivo sia identico alla dimostrazione ${ }^{48}$.

Anche in quanto fondamentale, la volontà di verità non produce, dunque, di per sé prove inconfutabili: il processo intellettivo può certo giungere a una dimostrazione, ma tale modalità, non è che un ambito entro il quale si costruisce l'intero processo dell'intelligenza che è sempre precedente qualunque possibile

43 HD, p. 277 (tr. it., p. 188).

44 Ibid.

45 In questo modo, conclude Zubiri, «la volontà di fondamentalità come attitudine è il principio unitario del processo intellettuale di Dio e dell'adesione personale a Lui in quanto persona vera»: HD, p. 278 (tr. it., p. 189).

46 Cfr. HD, pp. 281-293 (tr. it., pp. 191-199).

47 HD, pp. 294-298 (tr. it., pp. 200-203).

48 HD, p. 282 (tr. it., pp. 191-192). 
dimostrazione $e^{49}$. Del resto lo scopo ultimo del processo intellettivo verso il suo fondamento non trova il suo sbocco conclusivo in una dimostrazione perfetta e rigorosa dell'esistenza di Dio, in quanto fondamento ultimo della realtà. Anche qualora si giungesse a dimostrazioni totalmente concludenti e precise, la conoscenza e l'accesso pieno a Dio richiederebbe sempre un'opzione libera all'interno del processo intellettivo.

Questo è il motivo per il quale è sempre possibile che l'uomo «non solo non giunga a una giustificazione, ma sospenda anche la fede $»^{50}$. È il caso dell'agnosticismo: «consiste, naturalmente, nel poggiarsi sull'ignoranza della realtà di Dio: "Non so se esiste" ${ }^{51}$. Qui non viene negata la realtà di Dio, Dio è «qualcosa» di cui non si può conoscere nulla, poiché l'uomo è di per sé incapace di conoscere qualcosa di quella realtà: «intelligisce che è reale in un modo che non può essere conosciuto» ${ }^{52}$.

L'agnostico, dunque, cerca Dio ma non giunge a trovarlo. Il semplice fatto di stare nella realtà e di essere aperti al fondamento della stessa per la volontà di fondamentalità è una ricerca per l'uomo. E, tuttavia, è una ricerca che non trova la realtà cercata: «Da questo punto di vista, l'agnosticismo è frustrazione nella ricerca intellettiva» ${ }^{53}$.

Ignoranza, inconoscibilità e frustrazione sono i tre caratteri principali che sono alla base dell'opzione negativa alla fede, un'opzione reale che non annulla il processo intellettivo né lo chiude, poiché non annulla la volontà di fondamentalità.

Ciò che è evidente in questa forma di dispiegamento della volontà di fondamentalità è che il processo intellettivo non viene abbandonato: «la volontà di fondamentalità è essenzialmente volontà di ricerca» ${ }^{54}$. In nessun momento la ricerca di Dio nella fede è un salto nel vuoto: si tratta sempre di un'opzione, invece, radicata all'interno di un processo intellettivo nel quale la verità è sempre attuale.

Tuttavia, può esserci il caso in cui si giunga alla fondamentalità senza però avanzare nell'intelletto: è l'atteggiamento che Zubiri denomina in-differenza: «Sarebbe reale e costituirebbe effettivamente il processo intellettivo di un momento della realtà, però si tratterebbe di un processo che non giunge a quella differenza: sarebbe l'intellezione in-differente $»^{55}$. È vera intellezione, c'è come un assaggio di opzione che però si arresta nel processo intelletti-

Cfr. HD, pp. 282-284 (tr. it., pp. 192-193)

HD, p. 285 (tr. it., p. 194).

HD, p. 286 (tr. it., p. 194).

HD, p. 287. (tr. it., p. 195) Pesa qui la differenza tra «inteligir e conocimiento» in Zubiri. Inteligir è sempre apprendere qualcosa nella formalità di realtà, da sé, la conoscenza è «intelligire la sua realtà profonda, è intelligire in che modo è attualizzata nel suo proprio fondamento, in che modo è costituita "nella realtà come principio misurate». X. ZuBIRI, Inteligencia y razon, cit., p. 161.

53 Ibid.

54 HD, p. 288 (tr. it., p. 196).

55 HD, p. 290 (tr. it., p. 197). 
vo. Vale a dire, l'intellezione di Dio si interrompe prima di ogni differenza: chi si disinteressa è indifferente a un Dio ozioso o a un Dio fondamento. L'agnostico sospende la fede, l'indifferente sospende la conclusione intellettiva, decide di non andare più in là: non si tratta di sospendere l'adesione, quanto di sospendere l'intellezione. Sospensione e non cessazione, come subito si affretta a sottolineare Zubiri: «è un'intellezione in senso stretto della fondamentalità come ambito di un momento della realtà: sarebbe qualcosa di realmente in-differente ${ }^{56}$. L'indifferente non dice «non mi interessa se c'è o meno Dio» quanto esprime il non voler andare oltre la semplice verifica della sfera nella quale sono inesorabilmente gettato.

Ma «indifferenza intellettiva è "a un tempo" non-preoccupazione (despreocupación) $\aleph^{57}$ : scelgo di non preoccuparmi di quest'area di fondamentalità. $\mathrm{E}$, tuttavia, questa non-preoccupazione non è assenza di opzione, ma decisione di non occuparsi di questa realtà che fuoriesce dall'ambito del processo intellettivo nel quale sono lanciato ${ }^{58}$.

Chi si disinteressa, pertanto, del problema di Dio mantiene un processo intellettivo e un'opzione: l'ambito della fondamentalità viene inteso nella sua indifferenza, scegliendo di vivere senza alcuna preoccupazione. Disinteressarsi del problema della fondamentalità, del problema di Dio, esprime dunque una volontà di fondamentalità.

L'in-differenza e la non-preoccupazione indicano due aspetti di un'unica attitudine rispetto alla fondamentalità della vita: vivere abbandonandosi alla vita nell'indifferenza fondamentale. A rigore l'indifferente non vive senza fondamentalità, bensì vive in una indifferenza fondamentale: il "lasciarsi vivere" assume perciò un carattere positivo, anzi, per certi versi, è un'attitudine che viene assunta in omaggio alla vita. Il lasciarsi vivere e la volontà di vivere, conclude Zubiri «definiscono un'unica volontà, una volontà di vivere che potremmo chiamare penultima: è la penultimità della vita. È volontà di vivere, però lasciandosi condurre da quel che sarà il fondamento. Chi si disinteressa di Dio vive alla superficie di se stesso: la sua vita è costitutivamente penultima ${ }^{59}$.

$\mathrm{Al}$ di là delle considerazioni circa l'ultimità o penultimità della vita, ciò che è interessante è il carattere proprio della volontà di fondamentalità che non coincide mai con una mera volontà di verità teoretica quanto con una costitutiva volontà di vivere. Ed è questa volontà che si dispiega nel processo intellettivo e nell'opzione. In questo modo viene superato il pericolo di considerare l'accesso a Dio come mera conoscenza dello stesso Dio. È conoscenza e consegna di una realtà personale che costitutivamente vuole vivere, e anche la conoscenza di

$56 \quad$ Ibid.

57 HD, p. 291 (tr. it., p. 198).

58 «Mancanza di preoccupazione non equivale a non-opzione. Chi ignora, dicevo, sa in qualche modo che cosa ignora. Parimenti, chi non è preoccupato sente che, al di là della sua mancanza di preoccupazione, è latente la sorda presenza di ciò di cui non si preoccupa; pertanto, è sotterraneamente diretto verso di esso». HD, p. 291 (tr. it., p. 198)

59 HD, p. 293 (tr. it., p. 199). 
Dio in quanto tale non sopprime la volontà di vivere ma la accresce nella misura in cui l'uomo intelligisce e opta per Dio come fondamento per la propria vita.

\section{L'ateismo Radicale del XX Secolo}

In Uomo e Dio, la questione dell'ateismo, al pari dell'indifferenza e della non-preoccupazione (des-preocupación), è introdotta all'interno delle possibili attitudini dell'uomo che opta di non occuparsi del problema del fondamento, e sono - commenta Zubiri- «eo ipso vite senza volontà di fondamentalità ${ }^{60}$. Nell'ateismo interviene, poi, un'ulteriore peculiarità: le questioni che la vita pone sono problemi intra-vitali, anzi «nella sua interezza, la vita non pone per queste persone alcun problema: è quella che è e nient'altro [...] Di conseguenza, non è necessario parlare di opzione, ma neppure di volontà di fondamentalità: è la vita a-tea ${ }^{61}$. Dunque, alla mancanza di fondamentalità l'ateismo aggiunge anche l'assenza di opzione: l'ateo sembrerebbe non optare per alcunché. Di qui l'idea che tale attitudine possa essere considerata addirittura più originaria di qualunque altra "opzione" possibile: «L'ateismo sarebbe, pertanto, l'attitudine primaria, e ogni altra attitudine di riferirsi a Dio in qualsiasi forma (credenza, agnosticismo, mancanza di preoccupazione) avrebbe bisogno di giustificazione» ${ }^{62}$.

Sappiamo come l'autore di Uomo e Dio cercherà di risolvere la questione dell'originarietà dell'ateismo all'interno della problematicità e dell'intellezione della fatticità dell'ateo. Tuttavia, sembra utile soffermarsi —alla luce dei corsi inediti appena pubblicati da Antonio González e Esteban Vargas ${ }^{63}$ - su quanto il filosofo spagnolo si spinge a dettagliare all'inizio del corso, dedicato a L'uomo e il problema di Dio, rispetto al problema dell'ateismo, un ateismo radicale.

Zubiri prende avvio da due considerazioni — si ricordi che siamo negli anni nei quali più forte era diventato l'appello a un rinnovamento dell'esperienza religiosa $^{64}$ : da una parte, infatti, il porsi non nei termini di un rinnegamento di

60 HD p. 288 (tr. it., p. 196).

61 HD p. 294 (tr. it., p. 200).

62 Ibid.

63 Si tratta, come si evince dalla presentazione di Sobre la religión, di tre corsi tenuti da Zubiri nel 1965 e nel 1968, segnatamente «Il problema filosofico della storia delle religioni» tenuto a Madrid nel 1965, «Il problema della storia delle religioni, sempre del 1965 ma impartito a Barcellona, e «L'uomo e il problema di Dio», tenuto a Madrid nel 1968.

64 Sono gli anni del Concilio Vaticano II e i suoi effetti iniziano a notarsi nella società spagnola già sul finire del 1968. «La iglesia se abre a la modernidad rectificando muchos comportamientos anteriores: se ha suprimido el juramento antimodernista y el Índice de libros prohibidos; se quiere dialogar con el mundo moderno y se confía en la ciencia y el progreso; se defiende el anteriormente condenado ecumenismo y la libertad de religión; se revaloriza la Biblia y su exégesis; se autoriza la utilización en la liturgia de las diferentes lenguas y se impulsa el respeto de la iglesias locales y nacionales y a las llamadas teologías del Tercer Mundo»: Corominas J. -Vicens J. A., Xavier Zubiri. La soledad sonora, Taurus, Madrid 2006, p. 644. 
Dio, quanto dell'assenza del problema stesso di Dio e, dall'altra, il chiedersi non cosa significhi la realtà o irrealtà di Dio, quanto domandarsi se il termine Dio possa ancora avere un significato ${ }^{65}$. Ecco delineato ciò che Zubiri indica con il termine ateismo radicale. Un'attitudine che abbraccia tre differenti forme: l'ateismo vitale, l'ateismo politico-sociale e l'ateismo religioso.

Una prima forma di ateismo è quello «vitale»: ha a che fare non tanto con una negazione di Dio ma con una negazione del «problema» di Dio in quanto problema. Ciò che chiede l'ateo non è, innanzitutto, se si debba combattere o giustificare una credenza in Dio, quanto giustificare se vi sia un problema che chiamiamo Dio. In realtà, più che di ateismo si tratterebbe, secondo il filosofo basco, di non-teismo, una specie di eliminazione di Dio per emarginazione ${ }^{66}$.

La seconda forma di ateismo, denominata politico-sociale, costituisce l'ambito di negazione vero e proprio, e ha nel marxismo il suo punto culminante, tanto che tutta la trattazione di tale forma verrà analizzata da Zubiri seguendo tre punti caratterizzanti la posizione marxista rispetto al problema di Dio. In primo luogo, si prende in considerazione l'interpretazione storica che si dà del teismo, poiché il marxismo, contro la posizione di Feuerbach, ha sempre sostenuto che la credenza in un Dio abbia avuto un impatto storico positivo, a partire dal ruolo di giustificazione del male individuale e sociale così da condurre l'umanità a una sorta di rassegnazione o, se si vuole, di mesta oppressione rispetto al divino. Un'oppressione che, però, è stata interpretata non come una situazione statica, bensì come un cammino di liberazione: una sorta di riconoscimento del carattere costruttivo dell'accadere umano nella storia che deve essere liberato da ogni tipo di alienazione dell'umano in una realtà trascendente. Anzi il rimando alla trascendenza viene risolto, nell'interpretazione marxista, attraverso il riferimento al pensiero hegeliano e, in particolare, alla funzione della ragione che si erge a spirito assoluto. Solo che mentre nel sistema hegeliano si tratta di una posizione dialettica, nel marxismo si tratta di qualcosa di molto più tangibile $\mathrm{e}$ sperimentabile: ripensare la funzione del lavoro come fonte di tutto il progresso e di tutta la costruzione dell'uomo nel corso della storia. Grazie alla negazione del trascendente, l'uomo si concepisce creatore di se stesso, una realtà assoluta nel mondo, che trova nel lavoro la sua realizzazione. E sono proprio il mondo materiale e il lavoro gli elementi che limitano l'uomo nella sua realizzazione. Come realtà assoluta, infatti, l'uomo è essenzialmente e formalmente libero: «ma è una libertà vincolata ad altri due infiniti: da un lato, l'infinità della materia che è inesauribile (il mondo), dall'altro, l'infinità di una capacità produttiva dell'uomo, che è proprio la realtà umana $»^{67}$. La libertà è, dunque, possibile solo all'interno di questi due infiniti materiali che delimitano il farsi dell'uomo e rendono incompatibili l'essenza dell'umanità con un qualunque rimando a una

65 «Ahora bien, se nos dice, non solamente el problema de Dios non tiene realidad alguna como tal problema en la vida, sino que, además, el vocablo mismo de Dios, como concepto, carece de sentido». X. ZuBIRI, Sobre la religión, cit., p. 382.

66 Cfr. Ibid., p. 383.

67 Ibid., p. 385. 
trascendenza. "L'uomo, in quanto realtà assoluta, è incompatibile con la realtà di Dio» ${ }^{68}$ : è la negazione tematica di ogni possibile deismo.

Infine, l'ultima forma di ateismo detto «religioso»: di cosa si tratta? Per introdurre ciò che si intende con questa locuzione Zubiri fa riferimento a un testo di Le Dantec sull'ateismo che, però, non appare nella documentazione disponibile nella biblioteca dello stesso filosofo spagnolo ${ }^{69}$. Tuttavia, al di là dell'autore di riferimento ciò che interessa il filosofo è tracciare chiaramente il suo pensiero su questa forma di idea attraverso cui si vorrebbe addirittura «salvare» il cristianesimo proprio attraverso l'ateismo ${ }^{70}$.

«Il posto di Dio viene occupato da Cristo, ma un Cristo che non è più Dio, ma uomo. Non che Dio non esista all'interno di questa concezione. Non si tratta di questo: che esista o meno è assolutamente indifferente, poiché in nulla entra ormai nella vita dell'uomo» ${ }^{71}$.

Ecco dunque spiegato l'ateismo in questa ultima forma: una sorte di religione nella quale Cristo svolga il compito assegnatogli dalla filosofia moderna culminata in Kant ed Hegel: quello di un "novello» Socrate che riceve la sua importanza dagli insegnamenti e dai valori trasmessi. La realtà di Dio viene soppiantata dalla realtà pura e semplice della fede in Cristo: tecnicamente non si tratta — anche qui come nel caso dell'ateismo vitale — di una negazione di Dio, ma della morte della fede in Dio. È la teologia della «morte di Dio»: al suo posto rimane la fede in Cristo, un Cristo spogliato da ogni natura divina.

Al fine di avvalorare tale ipotesi Zubiri fa largo uso di alcuni tra i maggiori esponenti della teologia protestante del XX secolo: Karl Barth, Rudolf Bultmann e Paul Tillich, tutti esponenti di quella teologia dialettica che, pur con sfumature differenti, poggia l'intera esperienza cristiana nel messaggio trasmesso dalla parola di Cristo e dai suoi atti ${ }^{72}$, come se tutto il cristianesimo — aveva detto Agostino nel suo De trinitate- dipendesse dall'esempio di Cristo e non dalla grazia della sua persona ${ }^{73}$. Se in Barth, infatti, non vi è possibile alcuna fede fondata in un'esperienza reale dell'uomo, per Bultmann la fede cristiana, pur appoggiandosi all'avvenimento di Cristo, l'unica cosa che può trasmettere all'uomo è il suo

$68 \quad$ Ibid.

69 Gli stessi curatori del testo zubiriano sono costretti a congetture: L'athéisme di Le Dantec (Paris 1907) o La religion de l'athée di Jules Sageret (Paris 1922). Di quest'ultimo, in effetti, vi è nella biblioteca di Zubiri un testo del 1922 dal titolo Le système du monde des chaledéens à Newton. Invece, è possibile reperire un testo francese sull'ateismo, Athées mes frér, di Sertillanges (Paris 1944). Tuttavia, l'indicazione di Zubiri all'autore francese deve indurci a ritenere indubbia la conoscenza del testo di Le Dantec, almeno nelle sue linee generali.

70 Zubiri X., Sobre la religión, o.c., p. 386.

71 Ibid.

72 Detta della «crisi», la teologia dialettica accetta in maniera incondizionata il principio kierkegaardiano per il quale si dà come presupposta l'infinita differenza qualitativa tra il tempo e l'eternità, tra la storia e la religione, tra la cultura umana e la fede.

73 «Questo è l'orrendo e occulto veleno del vostro errore: che pretendiate di far consistere la grazia di Cristo nel Suo esempio e non nel dono della Sua Persona». Agostino d'Ippona, Opera incompiuta contro Giuliano, II, 146. 
messaggio vitale, «i valori e la dottrina della vita», come rileva Zubiri ${ }^{74}$. Infine, in Tillich, contro ogni possibile super-naturalismo di cui è intriso tanto cristianesimo, si fa largo l'idea che Dio non possa essere se non il fondamento del nostro stesso essere, "qualcosa che è nel "fondo" stesso della nostra realtà ${ }^{75}$, tanto da giungere a dover ripensare lo stesso concetto di Dio.

Ciò che accomuna tutti questi tre orientamenti è, dunque, secondo Zubiri, l'idea di una rottura tra realtà e fede: per poter liberare il Vangelo è stato necessario abbandonare l'uso del termine Dio. Non solo: con la teologia della morte di Dio si è andati oltre, cercando di ricondurre il cristianesimo a una tecnica di rassicurazione sociale, nella quale la comunità svolge un ruolo di primo piano, consolando l'uomo nel suo soffrire quotidiano e facendo leva sulla compassione di Cristo, spogliato da ogni riferimento alla sua natura divina: «un uomo che ha elevato in un modo eccezionale e unico nella storia la fiducia, la lealtà e l'amore ${ }^{76}$. Non è più il Gesù delle due nature, umana e divina, bensì quello di «due vocazioni»: una vocazione personale per la quale, attraverso l'idea di Dio, si vuole trasmettere alcuni valori, patrimonio dell'umanità, e una vocazione di consegna totale agli altri uomini. Ed è l'unione di tali due aspetti, e non l'unione ipostatica delle due nature, a costituire la realtà di Cristo. La morte di Dio rappresenta, pertanto, l'inizio di una cristologia atea.

«El hombre moderno desconoce a Dios como realidad. No puede hablar de Él, ni tan siquiera analógicamente. No puede sino vivir cristianamente, pero etsi Deus non daretur, como si Dios no existiera. A lo sumo, se nos dice, es posible que el hombre a lo largo de la historia tenía una esperanza de que eso que hemos declarado que no funciona en la vida, que no sabemos si existe o no, aparezca ciertamente en alguna forma. Es, si se quiere, la dimensión escatológica de la historia. Pero, como quiera que sea, se trata de una muerte de Dios, por lo menos por lo que afecta a las etapas históricas que estamos viviendo ${ }^{77}$.

L'uomo moderno, pertanto, pur vivendo cristianamente, vive senza Dio: persino il rimando al Cristo storico è privato di Dio: è un Cristo senza Dio, vittima della sua stessa umanità. Sembra rimanere sullo sfondo la grande domanda di Dostoevskij ne I demoni: "La fede si riduce a questo problema angoscioso: un uomo colto, un europeo dei nostri giorni, può credere, credere proprio, alla divinità del figlio di Dio, Gesù Cristo?» ${ }^{78}$.

$74 \quad$ ZuBIRI X., Sobre la religión, o.c., p. 387.

75 Ibid., p. 388.

76 Ibid.

77 Ibid., p. 389.

78 Dostoevskis F. M., I demoni; Taccuini per «I demoni», a cura di Lo Gatto E., Sansoni, Firenze 1958, p. 1011. Sembra fargli eco la seguente affermazione dell'allora cardinal Joseph Ratzinger: «La fede ha ancora in assoluto una sua possibilità di successo? [...] perché essa trova corrispondenza nella natura dell'uomo. Nell'uomo vi è un'inestinguibile aspirazione nostalgica verso l'infinito. Nessuna delle risposte che si sono cercate è sufficiente; solo il Dio che si è reso finito, per lacerare la nostra finitezza e condurla nell'ampiezza della sua infinità, è in grado di venire incontro alle domande del nostro essere. Perciò anche oggi la 
Non è più, dunque, una negazione metafisica della realtà di Dio, né tantomeno una speculazione su Dio: è un ateismo radicale, tale da assumere una superiorità mai espressa dagli ateismi classici, perché intende mutare ogni riferimento a Dio, riportandolo nella sfera dell'uomo, un uomo inteso non in una particolare attitudine, bensì in quanto uomo.

Ma è proprio tale radicalità che Zubiri cercherà di contestare, mettendo in dubbio innanzitutto l'originarietà di una tale posizione che vorrebbe indicare l'ateismo come attitudine primaria, la conditio possidentis, sulla quale il teista dovrebbe giustificarsi, mentre «teismo e ateismo sono due modi di concludere il processo intellettivo rispetto al problema del reale» ${ }^{79}$, e l'ateismo non è la soluzione di un problema ma un modo di intendere il potere del reale, un modo di interpretare la fondamentalità della vita come pura fatticità.

Da questo punto di vista ogni posizione atea non può che esprimersi in un'opzione: si opta per l'autosufficienza della vita, qualcosa che basta a se stessa, una vita che è così com'è, e nient'altro. "L'autosufficienza della vita è l'opzione per la fatticità del potere del reale» ${ }^{80}$. È un'opzione, una consegna, un'adesione alla fatticità della vita: è una fede nella fatticità. L'ateismo è, quindi, ultimamente fede atea, poiché — spiega Zubiri in un appunto inserito a mano nell'ultima redazione di Uomo e Dio- la fede è adesione formale a una persona in quanto vera, e l'ateo «aderisce formalmente alla propria realtà formale come unica e sufficiente realtà personale vera ${ }^{81}$. È fede verso se stessi.

In questo modo l'ateismo esprime sia il momento intellettivo rispetto al potere del reale, sia il momento optativo dell'autosufficienza: sono i due momenti di un'unica volontà di fondamentalità il cui atto ricade su un io che termina in se stesso. È il modo di essere assoluto dell'autosufficienza. Pertanto, la volontà di fondamentalità dell'ateo non può che interpretarsi come volontà di essere, di un essere relativamente assoluto. L'ateo è di per se stesso assoluto, perché, da una parte, è fondamento della sua propria realtà, dall'altra, accetta la sua relatività per la fatticità della vita. L'uomo aderisce al suo essere relativamente assoluto senza uscire dalla realtà di cui si è caricato il suo io. Ma al tempo stesso vuol essere se stesso, assoluto, vale a dire, non determinato da nient'altro che da se stesso. Desidera essere l'autore della propria vita, pur sapendo che l'orizzonte della vita non è stato creato da lui e che tale orizzonte è necessario affinché anch'egli possa realizzarsi.

Università degli Studi di Bari Aldo Moro

Paolo Ponzio paolo.ponzio@uniba.it

[Artículo aprobado para publicación en enero de 2019]

fede cristiana tornerà a trovare l'uomo». Ratzinger J., Fede, Verità, Tolleranza. Il cristianesimo e le religioni del mondo, Cantagalli, Siena 2003, pp. 142-143.

79 HD, p. 297 (tr. it., p. 202).

$80 \quad$ Ibid.

81 Ibid. 